\title{
Evaluation of the Taxonomic Relationship of Micrococcus cryophilus, Branhamella catarrhalis, and Neisseriae by Comparative Polyacrylamide Gel Electrophoresis of Soluble Proteins ${ }^{1}$
}

\author{
RICHARD H. FOX and DAVID E. MCCLAIN \\ Department of Biology, The American University, Washington, D.C. 20016
}

\begin{abstract}
Electrophoretic profiles of soluble proteins derived from seven strains of Branhamella catarrhalis, Neisseria perflava, Neisseria sicca, and the psychrophile, Micrococcus cryophilus, were compared. The profiles produced from the strains of Branhamella catarrhalis showed a marked similarity except for that of strain $\mathrm{Ne} 4$ (ATCC 23246) which was distinguishable from those of the other six strains. Species-specific profiles demonstrating no characteristic species-related banding patterns were obtained from the soluble proteins of $N$. perflava and $N$. sicca. The profile from $M$. cryophilus was likewise specific and species distinct from those of the other bacteria tested. Identification of natural taxonomic interrelationships between these bacteria as determined by the electrophoretic separation of proteins are discussed.
\end{abstract}

The problematical status in the taxonomy of the gram-negative cocci Neisseria catarrhalis $(1$, 5) and Micrococcus cryophilus (14) has been described, and a suggestion for their possible taxonomic relatedness has been proposed (16). Catlin and Cunningham (5) reported N. catarrhalis to be an atypical neisseria based on its deoxyribonucleic acid (DNA) base composition of $41 \%$ guanine plus cytosine $(\mathrm{G}+\mathrm{C})$ compared to $50 \% \mathrm{G}+\mathrm{C}$ for other neisseriae and by its negligible interspecies transformation frequencies. However, high transformation frequencies between strains of this species were reported (6). Later evidence demonstrated a phenotypic homogeneity among the strains of $N$. catarrhalis (1) and little hybridization of the DNA from this species with the DNA from other genetically related species of the genus $(11,12)$. These facts led Catlin (4) to propose that the currently recognized species $N$. catarrhalis be taxonomically relocated to the new genus Branhamella gen. nov., within the family Neisseriaceae. The type species is designated as B. catarhalis comb. nov. This designation will be used throughout this paper.

The psychrophile $M$. cryophilus has become a taxonomic enigma within the genus Micrococcus since Mazenec et al. (14) reported on the cytological characterization of this bacterium. This psychrophile was described as possessing a capsule, its cells arranged pre-

${ }^{1}$ This work was supported by a Faculty Research Award from the Office of Graduate Studies and Research of The American University to R.H.F. dominantly in pairs and tetrads, and displaying a gram-negative appearance in its cell wall ultrastructure. These properties were not shown to be characteristic for the type species, $M$. luteus. Recently, Sleytr and Kocur (16) described similar observations in the fine structure of $M$. cryophilus. Moreover, these investigators recognized a close similarity in the fine structure of $M$. cryophilus to that described earlier (10) for various neisseriae, but especially $B$. catarrhalis. Accordingly, it was proposed (16) that $M$. cryophilus be taxonomically reassigned among the nonsaccharolytic Neisseriaceae. This, together with similarities in DNA base contents of $41 \% \mathrm{G}+\mathrm{C}$ for both $M$. cryophilus (2) and $B$. catarrhalis $(1,3,5)$, was viewed as further evidence to advance this taxonomic shift.

The purpose of this study was to determine whether a natural taxonomic relationship exists between $M$. cryophilus, B. catarrhalis, or Neisseria spp. based on a comparative evaluation of the electrophoretic profiles of their soluble proteins. The close taxonomic relationship implied from a resemblance in the fine structure and $\mathrm{G}$ $+C$ contents of $M$. cryophilus and $B$. catarrhalis may possibly be expressed in similarities between their protein profiles.

\section{MATERIALS AND METHODS}

Organisms. The bacterial strains tested, their designations and sources are listed in Table 1.

Culture conditions. Starter cultures of each bacterium were grown to maximum stationary phase in 20 
$\mathrm{ml}$ of $2 \%$ tryptic soy broth (TSB, Difco) in 125-ml Erlenmeyer flasks. All cultures of the $B$. catarrhalis strains, $N$. perflava and $N$. sicca were incubated at 30 $\mathrm{C}$ on a New Brunswick Scientific gyratory shaker for 20 to $23 \mathrm{~h}$. $M$. cryophilus was incubated at $18 \mathrm{C}$ for 35 to $40 \mathrm{~h}$ on an Orbit Environ Shaker (Lab-Line Instruments) placed in a walk-in cold room. The temperature was maintained at a constant $18 \mathrm{C}$ by thermostatic control. An inoculum of $2 \mathrm{ml}$ of starter culture was used to seed 1 -liter batches of TSB in 2-liter Erlenmeyer flasks. These were incubated respectively in the same manner as the starter cultures. Cultures of the $B$. catarrhalis strains were harvested when a turbidity of 60 to 70 units (about $10^{8}$ cells $/ \mathrm{ml}$ ) was reached as measured in a Klett-Summerson photoelectric colorimeter (660-nm filter). $M$. cryophilus, $N$. perflava, and $N$. sicca cells were harvested when the turbidity of the cultures reached 80 to 90 units (about $2 \times 10^{8}$ cells $/ \mathrm{ml}$ ).

Preparation of soluble extracts. Cells were harvested by centrifugation at $8,000 \times g$ at $4 \mathrm{C}$. The sedimented cells were resuspended and washed three times with cold $5 \times 10^{-2} \mathrm{M}$ tris(hydroxymethyl)aminomethane (Tris)-hydrochloride buffer ( $\mathrm{T}$ buffer), $\mathrm{pH}$ 7.8. The final pellet was resuspended in $1 / 100$ of the original culture volume with $\mathrm{T}$ buffer containing the following: $5.7 \times 10^{-4} \mathrm{M}$ ascorbic acid, $6.4 \times 10^{-4} \mathrm{M}$ L-cysteine $\mathrm{HCl}$, and $0.4 \mathrm{M}$ sucrose (RT buffer), $\mathrm{pH} 7.8$ (8). The suspension was disrupted by sonic treatment at $6 \mathrm{~A}$ (Bronson model S-75 Sonicator) for $4 \mathrm{~min}$ at 0 C. The resulting cell lysate was centrifuged at 20,000 $X g$ for $2 \mathrm{~h}$ at $4 \mathrm{C}$. The protein content of the clarified supernatant was determined by the method of Lowry et al. (13), with bovine serum albumin as the standard. Portions of the concentrated extract were distributed to vials, sealed, and then frozen and stored at $-10 \mathrm{C}$ until used.

Electrophoresis. Stock solutions and the procedures for the preparation of $7 \%$ separating polyacrylamide gels and $2.5 \%$ stacking gels were essentially as described by the Canalco Corp. (1968, Chemical Formulations for Disc Electrophoresis, Canalco Corp., Rockville, Md.). The electrophoresis apparatus used was the model 3-1750 Buchler Polyanalyst and model

TABLE 1. Bacterial strains

\begin{tabular}{c|l|l}
\hline \multicolumn{1}{c|}{ Strain } & \multicolumn{1}{|c}{ Designation } & \multicolumn{1}{c}{ Source } \\
\hline Branhamella & $\mathrm{Nell}^{a}$ & ATCC 25238 \\
catarrhalis & $\mathrm{Ne4}$ & ATCC 23246 \\
& $\mathrm{Ne76}$ & ATCC 8193 \\
& $\mathrm{Ne} 20$ & ATCC 8176 \\
& Ne9 & Catlin \\
& (ATCC 25240) & \\
& Ne83 & Catlin \\
& (ATCC 25239) & \\
Neisseria perflava & MFAU & Mid-West \\
N. sicca & SC & Mid-West \\
Micrococcus & B57 & Mid-West \\
cryophilus & & ATCC 15174 \\
\hline
\end{tabular}

${ }^{a}$ Neotype strains.

$b$ Wesley Catlin, The Medical College of Wisconsin.

${ }^{c}$ Mid-West Culture Service, Terre Haute, Ind.
3-1155 Buchler Regulated Power Supply (Buchler Instruments, Fort Lee, N. J.). Gel columns were polymerized in glass tubes $75-\mathrm{mm}$ long with an inside diameter of $5 \mathrm{~mm}$. Proteins in the thawed concentrated samples were adjusted to a concentration of $150 \mu \mathrm{g} / 0.05 \mathrm{ml}$ with RT buffer, and then layered on top of the stacking gel. The space above the protein sample was filled with electrophoresis buffer, $5 \times$ $10^{-3} \mathrm{M}$ Tris and $4.8 \times 10^{-2} \mathrm{M}$ glycine, $\mathrm{pH} 8.3(8)$. Bromophenol blue tracking dye was added to the buffer in the upper bath of the electrophoresis apparatus. A constant current of $3 \mathrm{~mA}$ per gel tube was applied. The electrophoretic separation was terminated when the dye front marker migrated to a distance of about $60 \mathrm{~mm}$ into the separating gel. During the electrophoretic separation the temperature was maintained at $0 \mathrm{C}$ by circulating ice water through the water-jacketed lower bath chamber. The gels were removed from the glass tubes and stained for $45 \mathrm{~min}$ in amido black $(0.5 \%$ in $7 \%$ acetic acid [wt/vol] $)$. Destaining was accomplished by placing the stained gels individually into perforated plastic test tubes which were then suspended by hooks into 1-liter of $7 \%$ acetic acid. This solution was continuously circulated through a charcoal column. Destaining was completed within 12 to $15 \mathrm{~h}$.

\section{RESULTS}

Variations in the total migratory distances of the dye front markers were typical between gels in the same electrophoretic run, and appeared to be independent of the origin of the cellular extract applied to the gels. The intensity of the stained gels varied even though the protein concentration of each sample was standardized to $150 \mu \mathrm{g} / 0.05 \mathrm{ml}$. However, this factor did not limit or complicate the subsequent profile comparisons.

Figure 1 shows the typical electrophoretic profiles derived from the seven strains of $B$. catarrhalis and the neotype strain of $M$. cryophilus. In order to demonstrate the detail in these banding profiles and to make visible the distinct but faint bands, schematic diagrams were prepared (Fig. 2). The band mobilities $\left(M_{f}\right)$ expressed in these diagrams were calculated from the ratio of the distance moved by each band to the distance moved by the dye front marker. The profile shown for strain $\mathrm{Ne} 11$, the neotype strain, was used as the standard for comparison. It can be seen that there is a striking degree of overall similarity in the profile signatures between the strains of $B$. catarrhalis, with the exception of that from strain Ne4 which is dissimilar and clearly species distinct. Ne4 is seen to differ from Ne11 most prominently in the distribution of bands between the range $M_{f} 0.10$ to $M_{f} 0.40$. However, many common bands are seen in the region $M_{f} 0.40$ to $M_{f} 0.90$. 


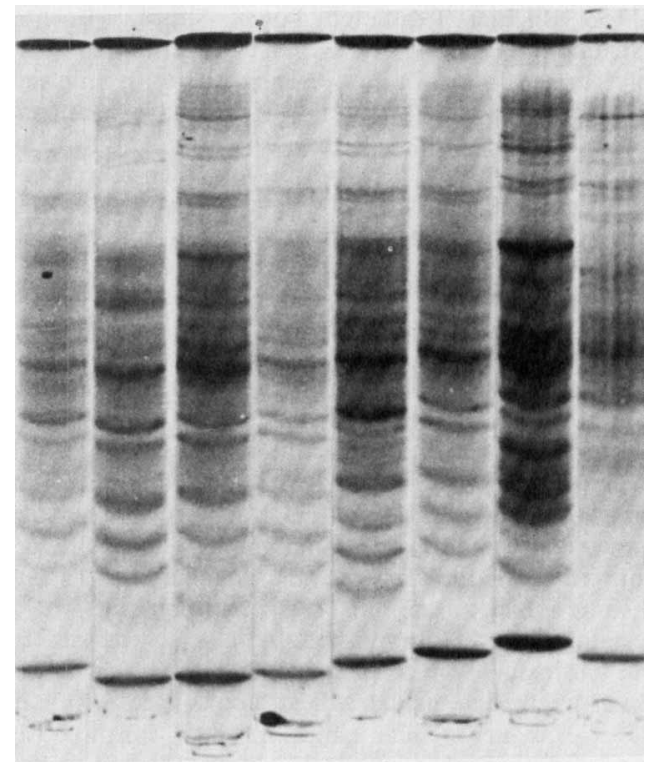

FIG. 1. Electrophoretic profiles of soluble proteins from seven strains of $B$. catarrhalis and $M$. cryophilus. (A) B. catarrhalis strain $\mathrm{Ne} 11 ;(B)$ strain $M W A U$; (C) strain Ne83; (D) strain Ne9; (E) strain Ne 76; (F) strain $\mathrm{Ne} 20$; (G) strain $\mathrm{Ne} 4$; and (H) $M$. cryophilus strain B57.
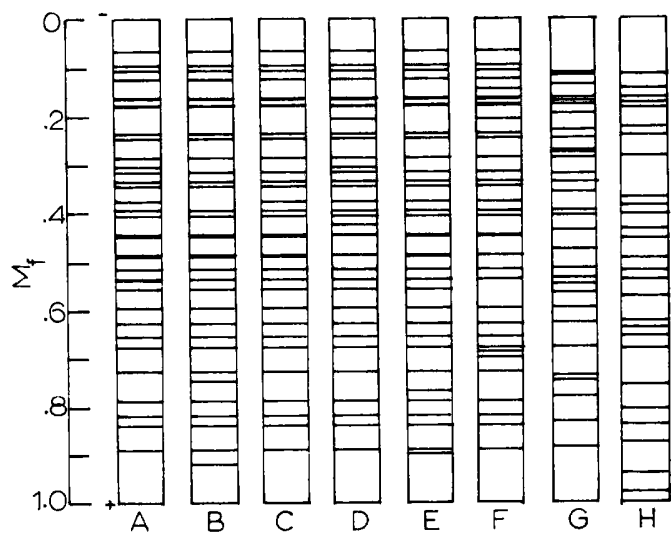

FIG. 2. Schematic diagrams of the electrophoretic profiles of soluble proteins from $B$. catarrhalis strains and $M$. cryophilus. (A) B. catarrhalis strain Ne11; (B) strain MWAU; (C) strain Ne83; (D) strain Ne9; (E) strain Ne76; (F) strain Ne20; (G) strain Ne4; and (H) M. cryophilus strain B57.

There is a definite species differentiation detected in the profile from B57 when compared to the profiles of both $\mathrm{Ne} 11$ and $\mathrm{Ne} 4$. Fewer similarities are seen between B57 and $\mathrm{Ne} 11$ than between $\mathrm{Ne} 11$ and Ne4. Both B57 and $\mathrm{Ne} 4$ exhibited band similarities in the region of slow mobility $\left(M_{f} 0.10\right.$ to $M_{f} 0.25$ ). With the exception of Ne4 and B57, the profiles of the other strains of $B$. catarrhalis show two identical bands at $M_{f} 0.07$ and $M_{f}$ 0.10 . The profile of B57 is further distinguished from the others shown in Fig. 2 by the absence of bands in the region $M_{f} 0.30$ to $M_{f} 0.37$.

Few band dissimilarities are seen upon comparing the profile of $\mathrm{Ne} 11$ with those from the $B$. catarrhalis strains, MWAU, $\mathrm{Ne} 83$, Ne9, $\mathrm{Ne} 76$, and $\mathrm{Ne} 20$ (Fig. 2). In general, the minor differences which do exist are detected as deletions and/or additions of one to five specific bands in any one of these gels.

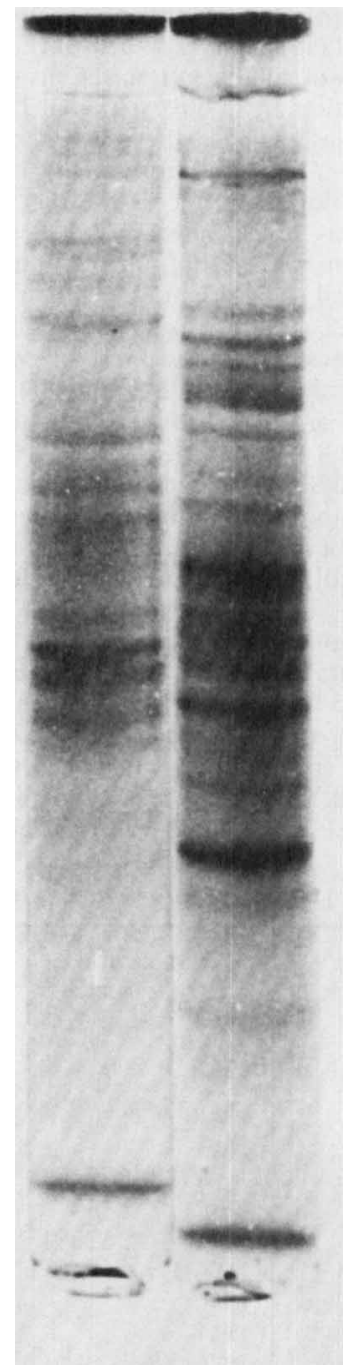

FIG. 3. Electrophoretic profiles of soluble proteins from two species of Neisseria. (A) $N$. perflava (PF); and $(B) N$. sicca $(S C)$. 


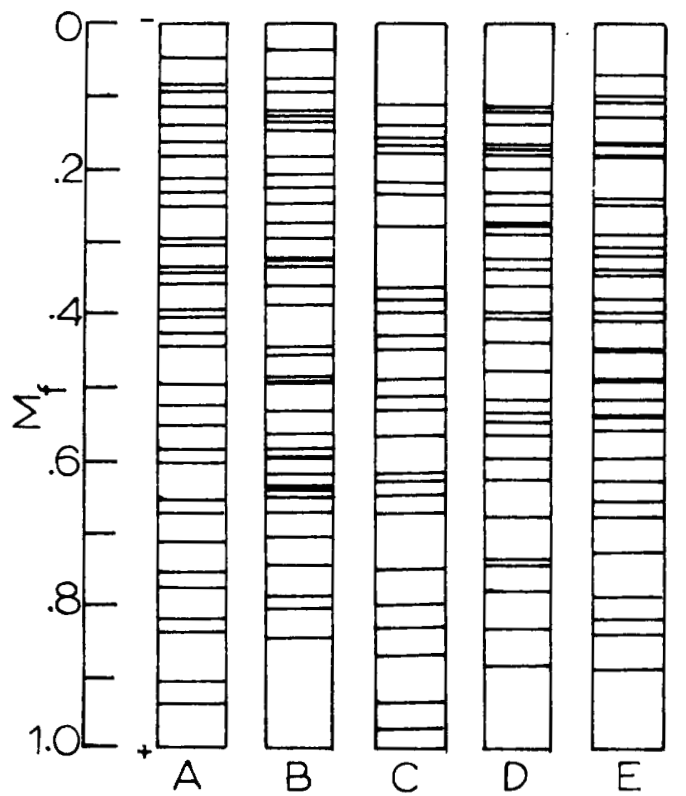

FIG. 4. Schematic diagrams of the electrophoretic profiles of soluble proteins from two species of Neisseria, B. catarrhalis strains and $M$. cryophilus. (A) $N$. perflava; (B) $N$. sicca; (C) $M$. cryophilus; (D) $B$. catarrhalis strain $\mathrm{Ne} 4$; and $(E) \mathrm{B}$. catarrhalis strain Ne11.

Moreover, the variations tend to occur exclusively in gel regions of both slow and rapid migrating minor bands. With few exceptions, bands in the region of intermediate mobilities $\left(M_{f} 0.32\right.$ to $\left.M_{f} 0.72\right)$ are virtually identical and characteristic of the species.

The protein profiles produced from $N$. perflava and $N$. sicca are shown in Fig. 3. The schematic representations of these are compared with those previously described for B57, $\mathrm{Ne} 4$, and Ne11 (Fig. 4). The profiles of the two species of Neisseria are as different from each other as they are from B57, $\mathrm{Ne} 4$, and $\mathrm{Ne} 11$. There are some scattered clusters of similar bands in the profiles of the two Neisseria species and detected in the regions $M_{f} 0.20$ to $M_{f} 0.25$ and $M_{f} 0.30$ to $M_{f} 0.40$. Some of these and other different clusters are seen to coincide with the profiles from $\mathrm{B} 57, \mathrm{Ne} 4$, and $\mathrm{Ne} 11$. There is a common band doublet in each of the profiles occurring in the region $M_{f} 0.33$ to $M_{f}$ 0.35 but not in the profile from B57.

\section{DISCUSSION}

The electrophoretic profiles resolved from multiple runs of the same and different soluble extracts prepared from the test organisms were highly reproducible. Reasons for the variations in staining intensities between gels separating identical quantities of proteins were not immediately obvious. Davies and Gottlieb (7) also observed this peculiarity and suggested that contaminating substances in the soluble extracts interfered with the accuracy of the quantitative protein assay. This explanation might perhaps be extended to account for the observations reported here. The idea that the proteins in the concentrated extracts used in this study were altered during the freezing and thawing prior to electrophoresis was excluded. No detectable differences could be shown in a specific profile or in its staining density upon electrophoresis immediately after preparation of the soluble extract or following its exposure to two freeze-thaw cycles. Demonstrable variations in the profiles did result after three or more cycles of freeze-thaw of the same sample. The standard procedure in this study was to separate electrophoretically proteins from oncethawed extracts and compare only those gels which were run simultaneously.

Profiles obtained from the $B$. catarrhalis strains were complex and species specific. Despite the few band variations exhibited it was possible to describe the profile from the neotype strain $\mathrm{Ne} 11$ as representative for the species. These results agree with the report of Baumann et al. (1) of genetic and phenotypic homogeneity for the strains of $B$. catarrhalis. An exception to this was seen in the profile from strain Ne4 (ATCC 23246) which was different from Ne11. The latter investigators reported that $\mathrm{Ne} 4$ could be differentiated physiologically from $B$. catarrhalis and suggested a rank for Ne4 of subgroup although it appears related to $B$. catarrhalis based on genetic evidence. The results shown here demonstrate that $\mathrm{Ne} 4$ is distinguishable from $\mathrm{Ne} 11$ and that a species designation would be appropriate. However, the generic assignment of $\mathrm{Ne} 4$ cannot be determined from the results of this study.

The profile from $M$. cryophilus (B57) was shown to bear no relationship to those from the B. catarrhalis strains including $\mathrm{Ne} 4$, or the two species of Neisseria. Indeed, the species specificity of the profile from $M$. cryophilus was as pronounced as that described between the latter two species. Detection of dispersed clusters of similar bands particularly in the profiles of $N$. perflava and $N$. sicca was interpreted as random occurrences rather than a real reflection of species relationships. Because of the numerous bands resolved in these gels it 
is likely that some proteins from the different species would have closely similar mobilities although such proteins are probably qualitatively dissimilar. One might have expected relatedness of species within the genus Neisseria when the comparison of their profiles exhibited a long linear sequence of similar bands, but not necessarily as extensive as seen between the closely related strains of $B$. catarrhalis. Other investigators $(7,9,15)$ have described similar results for the inability of the electrophoretic technique to identify taxonomic relatedness of species within and between different genera.

In certain respects the specificity shown to exist between the profiles of the two species of Neisseria contradict the genetic evidence $(5,11$, 12) for their taxonomic relatedness. The results suggest that the electrophoretic technique is also inadequate to confirm a natural taxonomic relatedness between $M$. cryophilus and the Neisseriaceae which is otherwise indicated on the grounds of close cytological and DNA base content similarities (16). Admittedly, evidence for taxonomic relatedness based on similarities from these latter types of phenotypic expressions is less substantial criteria than evidence obtained from DNA homologies.

In summary, the electrophoretic technique was shown to have limited value as a taxonomic tool, particularly when applied to the identification of species within and between genera. This limitation may be inherent in the fact that species profiles derived from whole cell lysates resolve such complex patterns as to preclude detection of definite relationships. The identification of strains of a species does not suffer from this limitation probably because of the greater overall genome homologies that exist between strains. In any event, this complication may be circumvented by the application of the electrophoretic technique to activity staining of certain enzymes and isozyme patterns chosen as appropriate taxonomic indicators.

From the results of this study, and in light of the limitations of the electrophoretic technique, we feel that there is insufficient information to reach a definitive conclusion bearing on the existence of a natural taxonomic relationship of $M$. cryophilus to the genus Branhamella or the genus Neisseria. Studies now in progress are examining some enzyme patterns of these species, and it is anticipated that resolution of this taxonomic problem will be forthcoming. It is concluded, however, that the presently designated $B$. catarrhalis strain ATCC 23246 (our strain $\mathrm{Ne} 4$ ) is distinct from this species. The elevation of Ne4 to a species taxon possibly within the genus Branhamella should be considered.

\section{LITERATURE CITED}

1. Baumann, P., M. Doudoroff, and R. Y. Stanier. 1968. Study of the Moraxella group. I. Genus Moraxella and the Neisseria catarrhalis group. J. Bacteriol. 95:58-73.

2. Bohacek, J., M. Kocur, and T. Martinec. 1969. DNA base composition and taxonomy of some micrococci. J. Gen. Microbiol. 46:369-376.

3. Bovre, K., M. Fiandt, and W. Szybalski. 1967. DNA base composition of Neisseria, Moraxella, and Acinetobacter, as determined by measurements of bouyant density in $\mathrm{CsCl}$ gradients. Can. J. Microbiol. 15:335-338.

4. Catlin, B. W. 1970. Transfer of the organism named Neisseria catarrhalis to Branhamella gen. nov. Int. J. Syst. Bacteriol. 20:155-159.

5. Catlin, B. W., and L. S. Cunningham. 1961. Transforming activities and base contents of deoxyribonucleate preparations from various neisseriae. J. Gen. Microbiol. 26:303-312.

6. Catlin, B. W., and L. S. Cunningham. 1964. Genetic transformation of Neisseria catarrhalis by deoxyribonucleate preparations having different average base compositions. J. Gen. Microbiol. 37:341-352.

7. Davies, F. L., and D. Gottlieb. 1973. Polyacrylamide gel electrophoresis of soluble proteins from several genera of the family Actinoplanaceae. Int. J. Syst. Bacteriol. 23:43-48.

8. El-Sharkawy, T. A., and D. Huisingh. 1971. Electrophoretic analysis of esterases and other soluble proteins from representatives of phytopathogenic bacterial genera. J. Gen. Microbiol. 68:149-154.

9. El-Sharkawy, T. A., and D. Huisingh. 1971. Differentiation among Xanthamonas species by polyacrylamide gel electrophoresis of soluble proteins. J. Gen. Microbiol. 68:155-165.

10. Girad, A. E., and B. J. Cosenza. 1970. A comparison of the fine structure of Micrococcus diversus, Neisseria catarrhalis and Neisseria sicca. Antonie von Leeuwenhoek J. Microbiol. Serol. 36:233-240.

11. Kingsbury, D. T. 1967. Deoxyribonucleic acid homologies among species of the genus Neisseria. J. Bacteriol. 94:870-874.

12. Kingsbury, D. T., G. R. Fanning, K. E. Johnson, and D. J. Brenner. 1969. Thermal stability of interspecies Neisseria DNA duplexes. J. Gen. Microbiol. 55:201-208.

13. Lowry, O. H., N. J. Rosebrough, A. L. Farr, and R. J. Randall. 1951. Protein measurement with the Folin phenol reagent. J. Biol. Chem. 193:265275.

14. Mazenec, K., M. Kocur, and T. Martinec. 1966. Electron microscopy of ultra-thin sections of Micrococcus cryophilus. Can. J. Microbiol. 12:465-469.

15. Rosendal, S. 1973. Analysis of the electrophoretic pattern of mycoplasma proteins for the identification of canine mycoplasma strains. Acta Pathol. Microbiol. Scand. Sect. B 81:273-281.

16. Sleytr, U., and M. Kocur. 1971. Structure of Micrococcus cryophilus after freeze-etching. Arch. Mikrobiol. 78:353-359. 